\title{
Source de l'énergie des marées: énergie cinétique de la Terre ou énergie thermique du Soleil?
}

\section{What causes tidal energy: \\ the Earth's kinetic energy, or solar heat energy?}

\author{
PAR R. GIBRAT
}

INGÉNIEUR-CONSEIL A E.D.F. POUR LES USINES MATÉMOTRICES

PRÉSIDENT DU COMITÉ TECHNIQUE DE LA SOCIÉTÉ HYDROTECHNIQUE DE FRANCE

\begin{abstract}
Vers 1952, l'état des travaux des astronomes, d'une part, et des spécialistes des marées, d'autre part, permettait de croire que la source de l'énergie des marées était dans la rotation de la Terre. Deputs, une discussion approfondie des observations astronomiques ne permet plus d'être assuré d'une accélération séculaire de la Lune et il a donc para possible de soutenir qu'en fait, le Soleil, par son énergie thermique, assurait un couple accélérateur compensant, pour la vitesse de rotation actuelle maintenue ainsi constante, le couple retardateur des marées. L'énergie marémotrice aurait ainsi sa source dans l'énergie solaire.

L'auteur, après une discussion de ces idées nouvelles, arrive d̀ la conclusion décevante qu'il est impossible aujourd'hui, devant les chiffres, de prendre position.
\end{abstract}

\begin{abstract}
Around 1952, astronomers and tide specialists were inclined to agree that the source of tidal energy was probably to be found in the Earth's rotation. Since then, however, a very thorough discussion of astronomical observation results has cast some doubts on the validity of the secular lunar acceleration assumption, and there now appear to be grounds for suspecting that, by virtus of its heat energy, the sun produces an accelerating couple which, for the present constant speed of rotation, compensates the time-setarding couple.

After discussing these new ideas, the Author reaches the disappointing conclusion that, on the strength of actuel figures, no definite opinion can yet be voiced on this question.
\end{abstract}

\section{I. - INTRODUCTION}

Dans notre article dans le Bulletin de la Société Française des Electriciens publié en mai 1953 (1) nous fixions comme source de l'énergie des marées, l'énergie cinétique de rotation de la Terre. Nous nous appuyions alors essentiellement

(1) R. Grbrat. - L'énergie des marées - Bulletin de la Société Française des Electriciens, n ${ }^{\circ} 29$, mai 1953, pages 283-332 (le passage visé ici est page 290). sur un article de $H$. Mineur (2) et sur les travaux de Jeffreys (3).

D'une part, en effet, les calculs hydrauliques

(2) H. Mreun. - Etude d'un concept fondamental de la science, le temps (C.N.R.S.) contribution de "Institut d'Astrophysique de Paris, série A, n" 106, février-mars 1951.

(3) JefFreys. - Tidal friction in shallow seas (Phil. Trans., 1920, page 239). 
de Jeffreys donnaient, pour la puissance moyenne dissipée par les marées sur le globe tout entier, environ 1,1 milliard de $\mathrm{kW}(4)$, l'essentiel étant fourni par les mers peu profondes et resserrées comme la Manche, la mer d'Irlande, la mer du Nord, le détroit de Behring surtout (70\% du total à lui tout seul) etc, le total des océans profonds Atlanticue, Pacifique, océan Indien, etc, n'utilisant à peine qu'un millième de l'énergie totale dissipée.

D'autre part, l'observation du mouvement de la Lune et la comparaison avec les résultats de la théorie de ces mouvements conduisaient à cette époque les astronomes à admettre un résidu d'accélération séculaire du moyen mouvement non expliqué par la Mécanique céleste. On mettait ainsi en évidence l'existence d'un certain ralentissement de la vitesse de rotation de la Terre, libérant une puissance de 1,4 milliard de $\mathrm{kW}$, retrouvant ainsi de façon très satisfaisante l'ordre de grandeur précédent.

Il apparaissait donc fort plausible d'admettre que l'énergie des marées était, par l'intermédiaire du frottement des eaux sur les fonds des mers, empruntée à l'énergie de rotation de la Terre et entrainait ainsi son ralentissement. L'énergie marémotrice était ainsi la seule, avec l'énergie nucléaire, à ne rien devoir à l'énergie calorifique du Soleil.

Ce tableau est aujourd'hui (début 1962) beau- coup moins net et nous avons pensé nécessaire sur une précieuse indication de M. Coulomb, directeur général du C.N.R.S., de refaire aussi soigneusement que possible le point. On ne semble pas avoir gagné beaucoup en précision dans le calcul des énergies dissipées et on a surtout détruit les idées reçues. Par contre, dans la $3^{\circ}$ édition (5) du célèbre ouvrage de Jeffreys (1952), il y a une petite note de quelques lignes faisant allusion à une idée de Holmberg (6) capable, si elle était vérifiée, de tout transformer en introduisant indirectement l'énergie thermique solaire et ainsi détruisant toutes les idées de Jeffreys sur le sujet. En 1956, Sir Spencer Jones (7), l'astronome anglais spécialiste des mouvements de la Lune, estime parfaitement possible, après les travaux de Brouwer (8) et van Woerkom (9), qu'en fait la vitesse de rotation de la Terre soit constante, ce qui rend vraisemblable les idées d'Holmberg. Cependant, ici aussi, une petite note dans le travail de Spencer Jones renvoie à un travail de Kertz (10) qui, en fait, parait détruire l'hypothèse d'Holmberg. L'ensemble vaut donc la peine d'être conté, car il fait appel à des notions très variées et rapproche des domaines lointains. Nous utiliserons largement les articles des astronomes ou géophysiciens, la consultation de leurs œuvres (références 5 à 10) étant malaisée. Mais les conclusions, par contre, n'engageront que nous.

\section{II. - FROTTEMENT DES MAREES}

Depuis notre article de la S.F.E. écrit d'après les premières éditions de Jeffreys, on a beaucoup écrit, mais la question ne s'est pas éclaircie, loin de là (11). Quelques précisions ou rappels sont cependant opportuns: la puissance moyenne dissipée par les marées sur le globe entier était, d'après Jeffreys, de 1,1 milliard de $\mathrm{kW}$ et ceci surtout dans les mers creuses; pour tous les océans, le total atteignait à peine un million de $\mathrm{kW}$. Bien que les vitesses soient de l'ordre du $\mathrm{cm} / \mathrm{s}$, le mouvement est cependant turbulent (nombre de Reynolds aux environs de 860). Pour les mers peu profondes, où les vitesses sont

\footnotetext{
(4) On notera qu'un milliard de $\mathrm{kW}$ équivaut à $10^{10} \mathrm{erg}$. seconde, unité utilisée par les astronomes.

(5) Jewrreys. - The Earth, $3^{\circ}$ édition, 1952.

(6) Holmbera. - Monthly Notices Royal Astron. Geophys., Suppl. 6, p. 325 (1952).

(7) Sir Spencer Jones. - Handbuch der Physik, t. XLVII, Geophysik I, p. 1 à 23 (1956).

(8) D. Brouwer. - Astronom. J, 57, 125 (1952).

(9) A.J.J. Van Woeriom. - Astronom. J., 58, 10 (1953).

(10) Kentz. - Handbuch der Physik, t. XLVIII, Geophysik II, p. 928-981 (1957).
}

beaucoup plus importantes, l'augmentation du taux par unité de surface proportionnellement au cube des vitesses contpense et bien au-delà la faiblesse des surfaces. Voici quelques chiffres de puissance moyenne, en millions de $\mathrm{kW}$, calculés par Jeffreys; nous mettons entre parenthèses les chiffres de Heiskanen (12).

\section{EUROPE}

Manche. ................ 55 (115)

Mer d'Irlande. . . . . . . . . . . . $30(20)$

Mer du Nord. . . . . . . . . . . . . . $35(\longrightarrow)$

(11) Une revuc assez complète des travaux récents se trouve dans l'excellent ouvrage de W. H. MuNK et G.J.F. MacDonald : The rotation of the Earth, Cambridge Univ. Press, 1960.

(12) Il faut en effet signaler cette étude peu connue de Heiskanen (1921) : Ueber den Einfluss des Gezeiten auf die «säkulare» et Accélération des Mondes. Ann. Acad. Scient. Fennicae, A. 18.1: il a obtenu pour la Terre entière 1,9 milliard de $k W$. Plus d'un tiers de son total est dû au frottement le long des côtes de grands océans, ce que Jeffreys a négligé. 
AsIE

\begin{tabular}{|c|c|}
\hline Mer Jaune. & $55(-)$ \\
\hline Mer d'Okhotsk. . . . . . . & 20 \\
\hline Mer de Béring. . . . . . . . . . & $750(480)$ \\
\hline étroit de Malacca........ & $55 \quad(90)$ \\
\hline tres mers. . . . . . . . . . . & $5(365)$ \\
\hline
\end{tabular}

AmériQue

Détroit d'Hudson. . . . . . . . . . 10

Détroit de Fox.............. 70

Baie de Fundy............... 20

Patagonie. ...............

Ơn notera à nouveau l'extraordinaire importance de la mer de Béring : deux tiers de toutes les pertes y trouvent place. Ceci permet d'avoir une idée de la précision des calculs, car une erreur très normale de $25 \%$ sur la valeur des courants dans cette mer suffit à modifier le montant total sur le globe de moitié de sa valeur. En fait, les deux auteurs partent de vitesses de deux nœuds et demi (Jeffreys) et deux nœuds (Heiskanen), vitesses observées sur les îles. II n'y a toujours pas aujourd'hui d'observations précises en nombre suffisant dans cette mer de $1000 \mathrm{~km}$ sur $1000 \mathrm{~km}$. Mais, de la dérive d'un brise-glace américain en 1958 (Northwind); de deux ou trois mesures faites dans la partie est et des observations d'ordre général faites par le sous-marin atomique «Nautilus» dans son voyage submergé au pôle, d'août 1958 , il semblè à Munk et à MacDonald que la limite supérieure des courants ne doit pas dépasser un nœud et que par suite la dissipation d'énergie dans la mer de Bering doit être de l'ordre de 8 millions de $\mathrm{kW}$, soit $1 \%$ des valeurs précédemment admises. C'est évidemment assez décourageant.

Par ailleurs, Jeffreys, faute de renseignements, n'a pu traiter le cas du passage arctique nordouest; de même, il y aurait au large de la Patagonie une surface très importante de mers peu profondes, de $500 \mathrm{~km}$ de large par 2000 de long, mais on connaît mal les courants, etc. Cependant Munk et MacDonald pensent que l'estimation de Heiskanen est correcte (observations du service hydrographique argentin). L'accord obtenu entre le chiffre total de 1,1 milliard de $\mathrm{kW}$ pour la puissance retardatrice et de 1,4 pour la puissance dégagée par le ralentissement de la Terre tel qu'on l'estimait au même moment, est donc très satisfaisant. Certains calculs récents doivent encore être signalés; le français Allard, dès 1951, avait calculé les pertes dans la Manche et trouvé 80 millions de $\mathrm{kW}$ en puissance moyenne annuelle; le canadien Mac Cellan (13) a calculé très soigneusement tous les aspects énergétiques dans la baie de Fundy et trouvé 30,9 millions de $\mathrm{kW}$. Tout ceci donne une bonne idée de la très faible précision à attendre des calculs genre Jeffreys ou de Heiskanen. On peut aussi essayer de calculer le chifire global par l'estimation du travail fait par la Lune et le Soleil, à l'aide des observations (amplitude el phase) de la marée. Un récent calcul de Groves et Munk (1959) cité dans la référence (11) donne 3,2 milliards de $\mathrm{kW}$ pour la Lune et 1,0 pour le Soleil. En fait, ce genre de calcul est peu précis à cause des points amphidromiques où la phase varie énormément. Nous laisserons au lecteur la responsabilité de choisir finalement un chiffre. Pour le moment, il nous paraît bien difficile de le faire.

On notera enfin, d'après les calculs de Jeffreys, que toute l'énergie existant dans la marée à un moment quelconque est dissipée en huit ou dix heures seulement; ce qui explique la faible influence des états antérieurs, mais rendait assez difficile une concentration de l'énergie globale des marées dans des mers comme celle de Béring, puisqu'il faut expliquer que toute l'énergie des marées du globe s'y concentre toutes les dix-huit heures, ce qui serait le cas si on acceptait le chiffre de Jeffreys.

\section{III. - ACCÉLÉRATIONS SÉCULAIRES DES MOUVEMENTS DE LA LUNE ET DU SOLEIL}

Commençons par un bref rappel historique : Halley (1695), par l'étude des observations, avait le premier indiqué que le mouvement de la Lune en longitude paraissait être accéléré :

$$
\mathrm{L}=a+b \mathrm{~T}+c \mathrm{~T}^{2}
$$

( $\mathrm{T}$ sic̀cle julien de 36525 jours). L'accélération serait donc $2 c$, mais les astronomes appellent traditionnellement «accélération séculaire du moyen mouvement $\gg c$. Nous nous conformerons ici aussi à cet usage fâcheux. Euler et Lagrange échouent dans leurs essais d'expliquer $c$ (env. $10^{\prime \prime}$ arc/siècle). Laplace annonce en 1787 : l'action moyenne du Soleil sur la Lune tend à diminuer l'attraction de la Lune vers la 'Terre et ainsi à faire décroître la vitesse angulaire. Une fois

(13) H.J. MacCellax. - Energy considerations in the Bay of Fundy system. J. Tish. Bal Canada, 15 (2), p. $135(1958)$ 
que l'on en a tenu compte, la vitesse angulaire reste constante. Mais cette action moyenne du Soleil dépend de l'excentricité de l'orbite de la Terre, excentricité qui décroil séculairement comme l'action des planètes sur la Terre, d'où une diminution régulière de l'action moyenne du Soleil, d'où lent accroissement du mouvement moyen de la Lune.

Laplace a trouvé ainsi $10^{\prime \prime}, 18 /$ siècle en excellent accord avec la valeur expérimentale. Mais en 1853, J. C. Adams a remarqué que Laplace supposait que la vitesse aréolaire de la Lune ne changeait pas. Adams a montré qu'il n'en était pas ainsi si l'excentricité n'était pas constante et qu'il $y$ avait donc un effet non compensé. Le chiffre est alors réduit à $5^{\prime \prime}, 70$, environ la moitié de la valeur observée. Il est d'ailleurs remarquable que les idées d'Adams ne furent pas acceptées en général (par exemple Le Verrier).

De là vint l'idée de rechercher un effet provenant d'une « retardation 》 séculaire de la rotation de la Terre. Kant avait décrit dès 1754 pour un concours de l'Académie de Berlin, concours présidé par Maupertuis, que l'action de la Lune en créant des marées dans les océans doit avoir un effet secondaire de retardation du mouvement de la Terre par frottement des marées. Il n'eut pas le prix. Laplace avait rejeté plus tard cette idée car d'après lui cela entrainerait une accélération des moyens mouvements des planètes. Or, celle-ci ne fut observée qu'en 1905 pour le Soleil (Lowell), puis plus tard pour Mercure et Vénus. Le frottement des marées apparut alors, écrit Spencer Jones, comme une vera causa de la retardation séculaire de la rotation de la Terre et Adams fut accepté.

Mais passons à l'examen des faits : même en nous bornant aux discussions des trente dernières années, ce n'est pas simple et, aujourd'hui encore, les plus éminents spécialistes se refusent à admettre que les observations astronomiques ne peuvent servir à trancher le débat (par exemple, Munk et MacDonald dans leur livre déjà cité de 1960).

Nous reproduisons ici en en modifiant l'ordre pour les rendre plus clairs, Ies raisonnements de Jeffreys; ils restent d'ailleurs toujours élémentaires :

a) Si la vitesse de rotation de la Terre $w$ diminue, au bout d'un temps $T$, la Terre sera en retard de $(1 / 2) \mathrm{T}^{2}(d \dot{w} / d t)$; une étoile passera donc au méridien trop tôt d'un temps ( $\mathrm{T}^{2} / 2 w$ ) $(d w / d t)$. Si nous tenons compte du moyen mouvement de la Lune $n$ par rapport aux étoiles, le changement du temps d'observation donnera un gain de la Lune sur sa position en angle de $n$ fois cette valeur, mais compte tenu de la vitesse de variation de son moyen mouvement $n$, l'effet apparent sera un gain de :

$$
\frac{1}{2} \mathrm{~T}^{2}\left(\frac{d n}{d t}-\frac{n}{w}\right)
$$

Par suite, l'accélération séculaire observée sera :

$$
\nu=\frac{1}{2}\left(\frac{d n}{d t}-\frac{n}{w} \frac{d w}{d t}\right)
$$

Même résultat pour le Soleil en accentuant les lettres convenables:

$$
v^{\prime}=\frac{1}{2}\left(\frac{d n^{\prime}}{d t}-\frac{n^{\prime}}{w} \frac{d w}{d t}\right)
$$

b) La loi d'attraction ( $M, m$ masses de la Terre et de la Lune, $c$ distance de la Lune à la Terre) donne :

$n^{2} c^{3}=f(\mathrm{M}+m) \quad f$ constante de gravitation.

Le moment angulaire de la rotation de l'ensemble Lune et Terre autour du centre de masse est $M m c^{2} n /(M+m)$. Jeffreys introduit la variable auxiliaire $y$ telle que :

$$
n=n_{0} y^{-3} ; \quad c=c_{0} y^{2}
$$

$n_{0}$ et $c_{0}$ sont les valeurs au temps initial. Au couple $(-N)$ agissant sur la rotation de la Terre due aux marées lunaires, correspond un couple $(+N)$ tendant à faire croître le moment angulaire précédent, soit :

$$
\frac{\mathrm{Mm}}{\mathrm{M}+\mathrm{m}} c_{0}^{2} n_{0} \frac{d y}{d t}=\mathrm{N}
$$

On aura de même avec le Soleil $\left(m^{\prime}, c^{\prime}\right.$ $g^{\prime}$ et $\mathrm{N}^{\prime}$ ) :

$$
\frac{\mathrm{M} m^{\prime}}{\mathrm{M}+m^{\prime}} c_{0}^{\prime} n_{0}^{\prime} \frac{d y^{\prime}}{d t}=\mathrm{N}^{\prime}
$$

car les différences des périodes des marées solaires et lunaires sont telles que les effets séculaires des marées solaires sur la Lune et des marées lunaires sur le Soleil sont nuls.

Enfin, si $\mathrm{C}$ est le moment d'inertie de la Terre autour de son axe, on a l'équation reposante :

$$
\mathrm{C} \frac{d w}{d t}=-\mathrm{N}-\mathrm{N}^{\prime}
$$

c) Il est maintenant facile d'exprimer $\nu$ et $v^{\prime}$ en fonction de $\mathrm{N}$ et $\mathrm{N}^{\prime}$, car :

$$
\frac{d n}{d t}=-\frac{3 n_{0}}{y^{4}} \cdot \frac{d y}{d t}
$$


d'où :

$$
y=-\frac{3}{2} \frac{\mathrm{M}+m}{\mathrm{Mm}} \frac{\mathrm{N}}{c_{0}{ }^{2} y^{4}}+\frac{n_{0}}{2 c w} \frac{\mathrm{N}+\mathrm{N}^{\prime}}{y^{3}} .
$$

Sur les quelques milliers d'années au plus sur lesquelles s'étendent les observations d'éclipses, on peut prendre $y=y^{\prime}=1$. En appelant $k$ le rapport actuel des moments de rotation de l'ensemble Terre-Lune autour de son centre de masse et de la Terre autour de son axe, soit :

$$
k=\frac{\mathrm{Mm}}{\mathrm{M}+\mathrm{m}} \frac{c_{0}^{2} n_{0}}{\mathrm{cw}}
$$

il vient pour la Lune :

$$
v=\frac{\mathrm{M}+m}{2 \mathrm{Mm} c^{2}}\left[k\left(\mathrm{~N}+\mathrm{N}^{\prime}\right)-3 \mathrm{~N}\right]
$$

et pour le Soleil :

$$
v^{\prime}=\frac{\mathrm{M}+m}{2 \mathrm{M} m c^{2}} \cdot \frac{n^{\prime}}{n} k\left(\mathrm{~N}+\mathrm{N}^{\prime}\right)
$$

le rapport du premier terme dans $v^{\prime}$ au second étant de l'ordre de $10^{-6}$ d'où la disparition de $\mathrm{M}^{\prime}$ et $c^{\prime}$ (masse et distance relative au Soleil). On voit que $d w / d t$ est proportionnel, mais de signe contraire, à l'accélération séculaire du soleil $v^{\prime}$. On notera finalement :

$$
\frac{\nu}{v^{\prime}}=\frac{(k-3 / k) . \mathrm{N}+\mathrm{N}^{\prime}}{\mathrm{N}+\mathrm{N}^{\prime}} \cdot \frac{n}{n^{\prime}}
$$

Actuellement on a :

$$
k=4,82 \quad \text { et }\left(n / n^{\prime}\right)=13,4 \text {. }
$$

d) Le taux de décroissance de l'énergie mécanique totale $\mathrm{E}$ du système Terre, Lune, Soleil doit être égal au travail des couples, d'où :

$$
-\frac{d \mathrm{E}}{d t}=\left(\mathrm{N}+\mathrm{N}^{\prime}\right) w-\mathrm{N} n-\mathrm{N}^{\prime} n^{\prime}
$$

l'action des marées lunaires par exemple est $N(w-n)$; elle est retardatrice, comme $w-n$ est positif, $\mathrm{N}$ est donc positif, donc $d v / d t$ est positif, la distance $c$ Terre-Lune croît et le moyen mouvement de la Lune décrôt. Il en est de même pour le Soleil, donc $d w / d t$ est négatif, la Terre subit un couple de ralentissement. e) Pour calculer numériquement le taux de ralentissement, Jeffreys suppose connue l'accélération séculaire de la Lune y (il la prend égale à $5^{\prime \prime}, 22$ ) et détermine le rapport $\mathrm{N} / \mathrm{N}^{\prime}$ des couples dus aux marées lunaires et solaires. Il obtient le rapport en faisant une hypothèse sur le frottement des marées : par exemple, avec un frottement proportionnel au carré de la vitesse, il obtient $\left(\mathrm{N} / \mathrm{N}^{\prime}\right)=3,4 ;-(d E / d t)$ est alors environ $1,39.10^{19} \mathrm{ergs} / \mathrm{s}$, soit 1,4 milliard de kilowatts.

Nous avons vu plus haut que les calculs directs au moyen des équations de l'hydraulique des pertes d'énergie dans les mers, surtout celles peu profondes, avaient donné à divers auteurs vers 19301,1 milliard de kilowatts, ce qui représenterait $80 \%$ du chiffre total; comme, d'une part, Jeffreys lui-même estimait que le calcul des frottements pouvait comporter $50 \%$ d'erreur, d'autre part qu'il pouvait y avoir, comme l'a indiqué H. U. Sverdrup, à tenir compte des surfaces recouvertes de glace de l'Arctique et de l'Antarctique, on comprend donc que de nombreux auteurs, par exemple Defant encore, en 1957 dans son grand article du «Handbuch der Physik», t. XLVIII, s'en tenaient à cette explication.

La difficulté essentielle provient de ce que le rapport $\left(\mathrm{N} / \mathrm{N}^{\prime}\right)=3,4$ donne pour le rapport des accélérations séculaires $\nu / \nu^{\prime}$ une valeur de 7,2 .

Jeffreys a aussi fait le calcul de $\mathrm{N} / \mathrm{N}^{\prime}$ en admettant un frottement proportionnel à la vitesse, il a obtenu un rapport des couples de 5,1, qui conduit pour $y / y^{\prime} \dot{a} 6,3$. Enfin, on notera que la valeur minimale du rapport des accélérations séculaires serait de 5,0 pour $\mathrm{N}^{\prime}$ nul, c'est-à-dire pour l'absence de frottement des marées solaires. Or, comme on va le voir, aucune des valeurs du rapport des accélérations séculaires que l'on pouvait tirer de l'observation n'atteint, et de loin, cette valeur de 5,0 cependant extrême. Ceci a été pendant longtemps une énigme et explique pourquoi on a dû chercher plus loin. Mais avant d'exposer ces recherches, expliquons maintenant la position actuelle des astronomes.

\section{IV. - VALEURS OBSERVEES DE $\vee$ et $v^{\prime}$}

a) Dès 1878, Newcomb, par l'étude approfondie des tables de Hansen (1851) et des observations de la Lune (depuis 1675), avait montré qu'il y avait, en dehors des termes dus aux diverses attractions, un terme inexpliqué appelé par lui «Great empirical term», d'une période de 260 années. Brown, en 1919, avait publié, après une nouvelle discussion complète de la théorie de la Lune, introduisant près de deux mille termes, des tables comprenant, en plus de la part calculable due à la gravitation, le grand terme empirique toujours inexpliqué, mais non l’influence des marées. Le Nautical Almanac, depuis 1923 , donne d'après ces tables la place de 
la Lune dans le ciel. Or, depuis cette époque, malgré la présence $d u$ «Great empirical term », on constate des différences variables entre la table et les observations. Comme il est maintenant certain que la théorie de la Lune est pratiquement parfaite, du point de vue de la Mécanique céleste, il faut essayer d'expliquer les différences par des variations dans la vitesse de rotation de la Terre.

b) Les anciennes observations utilisent les plus vieilles éclipses connues; une des plus anciennes utilisables est de 683 avant JésusChrist (nous citerons un texte célèbre sur une éclipse à propos de Assur Isid Raki Rabe de la cité de Gozon «Dans le mois de Silvan, l'année de l'insurrection dans la cité d'Assur...»); la plus importante des éclipses utilisées fut observée par Hipparche le Rhodien en 128 avant Jésus-Christ. Voici la citation : «Une fois le Soleil parut totalement éclipsé à Hellespont, tandis qu'à Alexandrie il ne l'était qu'aux quatre cinquièmes de son diamètre.» Deux éclipses satisfont à la condition : 309 avant Jésus-Christ et 128 avant Jésus-Christ. On admet 128 parce qu'il est plus normal qu'Hipparche pense à une observation contemporaine. Agathoclès, Archilochus, Archimède, Palégon, Pindare, Plutarque, etc., sont ainsi successivement utilisés. Mais, hélas, les observations chinoises ou de l'Amérique centrale (Maya, par exemple), n'ont pas attiré l'attention des astronomes; la discussion la plus complète a été faite par de Sitter (1927) (14); elle a donné en dernier lieu les résultats suivants, avec indication de l'erreur moyenne :

$$
\begin{aligned}
& \text { Lune } v=5^{\prime \prime}, 22 \pm 0^{\prime \prime}, 30 \\
& \text { Soleil } v^{\prime}=1^{\prime \prime}, 80 \pm 0,16 \\
& \text { rapport } v / v^{\prime}=2,9
\end{aligned}
$$

ce rapport, on le voit, est bien différent des rapports calculés plus haut.

En 1939, Sir Spencer Jones a pris pour point de départ la fluctuation $\mathrm{B}$ des mouvements de la Lune définis par :

$$
\mathrm{B}=\text { observation }-\mathrm{C}
$$

avec $\mathrm{C}=$ tables de Brown

$-10,71 \sin \left(140^{\circ}, 0^{\mathrm{T}} \mathrm{T}+240^{\circ}, 7\right)$

$$
+5^{\prime \prime}, 22 \mathrm{~T}^{2}+12^{\prime \prime}, 96 \mathrm{~T}+4^{\prime \prime}, 65
$$

d'où $B+$ termes du second degré

= observation - termes mécanique céleste.

(14) W. de Sitrer. - Bull. Astronom. Inst. Netherlands, 4,21 (1927). Afin de concilier ses résultats avec l'explication utilisant le frottement des marées, il suggérait que celui-ci était autrefois (avant J.C.) deux fois plus important, mais il est difficile de se l'expliquer.
$\mathrm{T}$ mesure le temps en siècles commencant à 1900.0. Le terme en sinus enlève des tables de Brown le «Great empirical term», dont il faut done saluer la disparition définitive, ce qui est tout à l'honneur des astronomes; celui en $\mathrm{T}^{2}$ incorpore $v$, les deux autres sont de simples corrections permettant de se ramener à l'instant initial (1900.0). Le grand intérêt de cette décomposition réside dans le fait que le terme $B$ représentant les variations périodiques saisonnières ou non de rotation de la Terre convenablement multiplié par les rapports des moyens mouvements, doit se retrouver dans les observations du Soleil, de Mercure, de Vénus, etc., alors que les termes en $\mathrm{T}^{2}$ se déduiront pour Mercure et Vénus, non de celui de la Lune $v$, mais de celui du Soleil $v^{\prime}$. En d'autres termes, les différences en longitude des divers astres entre les observations et les calculs de la Mécanique céleste devraient être de la forme :

Lune : $\quad a+b \mathrm{~T}+5^{\prime \prime}, 22 \mathrm{~T}^{2}+\mathrm{B}$

Soleil : $\quad a^{\prime}+b^{\prime} \mathrm{T}+\frac{n}{n^{\prime}} v^{\prime} \mathrm{T}^{2}+\mathrm{B}$

Mercure: $a^{\prime \prime}+b^{\prime \prime} \mathrm{T}+v^{\prime} \frac{n^{\prime \prime}}{n^{\prime}} \mathrm{T}^{2}+\frac{n^{\prime \prime}}{n} \mathrm{~B}$

Vénus : $\quad a^{\prime \prime \prime}+b^{\prime \prime \prime} \mathrm{T}+\mu^{\prime} \frac{n^{\prime \prime \prime}}{n^{\prime}} \mathrm{T}^{2}+\frac{n^{\prime \prime \prime}}{n} \mathrm{~B}$

Or, les observations modernes, c'est-à-dire en fait depuis 1680 pour la Lune, le Soleil (déclinaison) et Mercure, depuis 1830 pour Vénus et le Soleil (ascension droite) et cela jusqu'en 1940, sont représentées de façon très satisfaisante par ces équations. Sir Spencer Jones a donc donné ainsi une solution définitive au problème de la Lune renvoyant les difficultés sur la rotation de la Terre, mais la valeur correspondante calculée ainsi pour l'accélération séculaire $v^{\prime}$ (Soleil) est $1^{\prime \prime}, 23 \pm 0^{\prime \prime}, 04$, nettement différente de celle donnée plus haut par de Sitter.

c) Sir Spencer Jones explique cette discordance entre les résultats de Sitter appuyés sur plusieurs millénaires et les siens appuyés sur un petit nombre de siècles, par l'impossibilité de séparer l'accélération séculaire et les termes de fluctuation B. Car la quantité $B$ a été définie de façon à rendre égales ses valeurs extrêmes positive et négative entre 1675 et 1925 . Si on connaissait la vraie accélération de la Lune, les fluctuations seraient différentes des valeurs prises pour B. Par exemple, si on prend $v=5,22+s$ suivant une idée semblant remonter à T. E. Sterne en 1935, B devient $\mathrm{B}^{\prime}=\mathrm{B}-s \mathrm{~S}, \quad \mathrm{~S}$ étant de la forme $(\mathrm{T}-a)$ $(\mathbf{T}-b)$ de façon à être nul à deux époques 
choisies arbitrairement. Les termes intéressants pour le Soleil, la Lune et Mercure gardent la même forme :

$$
\begin{gathered}
\left(v^{\prime}+\frac{n^{\prime}}{n} s\right) \mathrm{T}^{2}+\frac{n^{\prime}}{n} \mathrm{~B} \\
\frac{n^{\prime \prime}}{n^{\prime}}\left(v^{\prime}+\frac{n^{\prime}}{n} s\right) \mathrm{T}^{2}+\frac{n^{\prime \prime}}{n} \mathrm{~B}^{\prime} \\
\frac{n^{\prime \prime \prime}}{n^{\prime}}\left(v^{\prime}+\frac{n^{\prime}}{n} s\right) \mathrm{T}^{2}+\frac{n^{\prime \prime \prime}}{n} \mathrm{~B}^{\prime}
\end{gathered}
$$

Or on peut déterminer $s$ en remarquant qu'il est compréhensible que la dissipation d'énergie par les marées ait pu changer pendant les derniers millénaires, par exemple avec une variation du niveau de la mer, mais qu'il est raisonnable d'admettre que le rapport des accélérations séculaires Lune, Soleil est resté constant, d'où :

$$
\frac{v}{v^{\prime}}=\frac{5,22 \pm 0,30}{1,80 \pm 0,16}=\frac{5,22 \pm 0,30+s}{1,23 \pm 0,04+0,0747 s}
$$

ce qui détermine $s$ :

$$
s=-2^{\prime \prime}, 11 \pm 0^{\prime \prime}, 57
$$

les accélérations séculaires moyennes déduites des observations des derniers siècles seraient donc :

$$
\begin{aligned}
& \text { Lune }>=3^{\prime \prime}, 11 \pm 0^{\prime \prime}, 57 ; \\
& \text { Soleil }>^{\prime}=1,07 \pm 0,06 ;
\end{aligned}
$$

rapport $y / v^{\prime}$ inchangé 2,9 . Ainsi se trouveraient utilisées à la fois et sans contradiction les vieilles observations et les modernes.

d) Mon histoire n'est malheureusement pas terminée, car la courbe représentant les variations de longitude de la Lune avec le temps a de petits changements de pente brutaux (changement correspondant à des variations de longueur du jour de 4 ou 5 milli-secondes en quelques mois). Les astronomes contemporains, après Brouwer (1952), les assimilent aux courbes d'erreur des pendules à balancier, bien connues maintenant que l'on dispose de pendules à quartz très régulières. Certains auteurs, Munk et Révelle (15) les expliquent soit par des irrégularités dans le couplage électromagnétique du manteau formant la croûte terrestre avec le noyau liquide, soit par des variations stochastiques dans l'altitude des divers continents. Nous ne nous attar-

(15) Munk et Revelde. - M.N. Geophys., Suppl. 6, 331 (1952). derons pas. Ce n'est pas notre sujet. Mais ce qui est très grave, c'est que l'effet cumulé de ces petits changements se traduit par une incertitude beaucoup plus forte sur la vraie valeur de l'accélération séculaire.

Van Woerkom, dans son travail déjà cité de 1953, a étudié de façon systématique les propriétés statistiques des séries cumulatives de $n$ nombres choisis au hasard et a démontré, à laide de la théorie des chaines de Markofi, que leurs premières et deuxièmes sommes ont des fluctuations de lordre de $\sqrt{n}$ et $(\sqrt{n})^{3}$. L'amplitude des fluctuations de la Lune et des planètes doit donc varier comme la puissance trois-demis de la durée des observations utilisées, si l'on admet que l'accélération subit des variations "stochastiques» comme celles que produirait une suite de nombres pris au hasard. Brouwer avait pu utiliser ces résultats dès 1952 après avoir vérifié que les faits observés pour la Lune étaient bien en agrément avec eux, la dérivée de la courbe de fluctuations des dernières 130 années ressemblant bien à celle qui se déduirait d'une suite de nombres pris au hasard.

Brouwer fixe finalement, en tenant compte de tout cela, les valeurs suivantes de $v$ et $v^{\prime}$ :

$$
\begin{aligned}
\text { Lune } v & =2^{\prime \prime}, 2 \pm 9^{\prime \prime}, 5 \\
\text { Soleil } v^{\prime} & =1^{\prime \prime}, 01 \pm 0,70
\end{aligned}
$$

La valeur des écarts moyens ne permet donc pas d'être certain qu'il y a une accélération séculaire positive de la Lune, et celle du Soleil reste douteuse; aussi on ne peut faire rejeter sans examen l'idée que la vitesse de rotation de la Terre serait constante, car nous avons vu plus haut que l'accélération de rotation de la Terre est proportionnelle, mais de signe contraire, à $v^{\prime}$; les chiffres déduits des observations anciennes s'expliquent maintenant grâce à l'effet accumulé des modifications dues au hasard : l'erreur moyenne pour la Lune est de plus de quatre fois la valeur la plus probable; le rapport des accélérations perd donc toute signification; la destruction de l'édifice bâti durant les siècles par les astronomes est maintenant complète. Les études statistiques de ce genre se prolongent actuellement grâce aux méthodes d'analyse des observations par spectre de fréquences, méthodes certainement très puissantes. Il est remarquable cependant de noter que Munk et MacDonald dans (11) tout en exposant et discutant les travaux de Brouwer et van Woerkom, ne retiennent pas le résultat sur l'erreur moyenne pour l'accélération séculaire, résultat cependant capital. 


\section{V. - HYPOTHESE DE HOLMBERG-KELVIN}

a) Comme nous l'avons écrit dans l'introduction, dans les notes qui terminent l'édition du grand ouvrage de Jeffreys (1952), exactement à la dernière page, on trouve ceci sous la numération $8.05 a:$ : La difficulté provenant dı rapport des accélérations peut être expliquée par un résultat de Kelvin. Il y a une marée solaire semi-diurne dans l'atmosphère, d'origine thermique, et l'attraction du Soleil sur cette marée crée un couple accélérateur qui n'est pas négligeable. Si on en tient compte, on peut rapprocher le rapport de la valeur désirable. Le problème est étudié dans un article à venir de E. R. R. Holmberg. » C'est précisément l'article que nous avons référencé en note (6).

Sir Spencer Jones, dans son article de 1956, admet comme certain que la pression barométrique présente une variation importante semidiurne dont la phase est constante sur le globe. Il rappelle que, dès 1882 , lord Kelvin avait suggéré de tenir compte de la coincidence entre la moitié de la période de rotation de la Terre et la période propre de résonance de l'atmosphère terrestre, la phase de cette variation (maximale dans les deuxièmes moitiés de la matinée et de la soirée 10 heures et 22 heures) étant telle que l'attraction $\mathrm{du}$ Soleil sur cette marée atmosphérique crée un couple accélérateur sur la Terre. Personne n'avait tenu compte de cette suggestion jusqu'au moment où Holmberg l'a reprise; il observe que le maximum des variations diurnes de température a lieu l'après-midi et celui de la variation diurne de pression dans la deuxième partie de la matinée, et en déduit qu'un certain travail est fait par l'atmosphère. Ce travail entretient une variation de pression qui empêche les maximums semi-diurnes de la pression de glisser dans les premières moitiés de la matinée et de la soirée sous l'action du frottement de l'atmosphère sur la surface terrestre. L'énergie mécanique correspondant au couple accélérateur aurait ainsi sa source dans l'énergie thermique du Soleil par un cycle analogue à celui de la machine à vapeur. Tout cela, avouons-le, n'est pas très clair.

Avec les données de Simpson (16) sur la variation semi-diurne :

$$
p=1250 \cdot \sin ^{3} \theta \cdot \cos \left(2 t+64^{\circ}\right) \mathrm{dyn} / \mathrm{cm}^{2}
$$

(16) G. C. Simpson. - Quart. J. Roy. Mat. Soc., 44, 1 (1918). Le couple correspondant à la marée lunaire atmosphérique est au contraire décélérateur, mais de l'ordre de $5 \%$ du couple accélérateur dù à la marée solaire atmosphérique. $\theta$ étant la colatitude, Holmberg trouve, indépendamment de toute théorie sur les marées atmosphériques, un couple de $3,7.10^{22} \mathrm{dyn} / \mathrm{cm}$ correspondant à une puissance de 0,22 milliard de kilowatts, à comparer à la puissance de frottement des marées de Jeffreys 1,1 milliard de kilowatts. Holmberg, poussant très loin son idée, s'est alors demandé, étant donnée la marge d'erreur qu'il est possible d'attribuer aux calculs de Jeffreys, si les deux couples ne pourraient pas être égaux, ce qui expliquerait la coïncidence actuelle entre la période de rotation de la Terre et la période propre de l'atmosphère. La Terre se serait ainsi ralentie progressivement par le frottement des marées jusqu'au moment où la moitié de sa période de rotation serait devenue proche d'une période de résonance de la marée atmosphérique. La marée atmosphérique aurait augmenté progressivement, accroissant le couple accélérateur. Ainsi, la vitesse de rotation serait désormais maintenue constante et tout emprunt d'énergie par une usine marémotrice à l'énergie cinétique de la Terre serait aussitôt compensé par un apport d'énergie solaire. La véritable source de l'énergie marémotrice deviendrait, comme le charbon ou l'énergie hydraulique, l'énergie thermique du Soleil.

Rien, comme nous l'avons vu plus haut, dans les valeurs de $v$ et $v^{\prime}$ déduites des observations ne peut plus faire rejeter l'idée d'une rotation constante de la Terre.

b) De plus l'hypothèse d'Holmberg est très satisfaisante si on cherche à trouver un rapport correct des accélérations séculaires $v$ et $v^{\prime}$.

c) Introduisons en effet avec Sir Spencer Jones dans les calculs de Jeffreys le couple accélérateur $N^{\prime \prime}$ de Holmberg dû̀ à la marée solaire semidiurne de l'atmosphère, d'origine thermique. Les équations deviendront :

$$
\begin{gathered}
\frac{d w}{d t}=\mathrm{N}^{\prime \prime}-\mathrm{N}-\mathrm{N}^{\prime} \\
\nu=\frac{\mathrm{M}+m}{2 \mathrm{M} m c^{2}}\left[k\left(\mathrm{~N}+\mathrm{N}^{\prime \prime} \cdots \mathrm{N}^{\prime \prime}\right)-3 \mathrm{~N}\right] \\
v^{\prime} \frac{\mathrm{M}+m}{2 \mathrm{M} m c^{2}}\left[k\left(\mathrm{~N}+\mathrm{N}^{\prime}-\mathrm{N}^{\prime \prime}\right) \frac{n^{\prime}}{n}\right]
\end{gathered}
$$

l'équation de l'énergie reste valable sans changement, car il ne s'agit ici que d'énergie mécanique :

$$
-\frac{d \mathrm{E}}{d t}=\left(\mathrm{N}+\mathrm{N}^{\prime}\right) w-\mathrm{N} n--\mathrm{N}^{\prime} n^{\prime} .
$$


Spencer Jones pose à ce moment :

$$
\mathrm{N}^{\prime \prime}=\lambda\left(\mathrm{N}+\mathrm{N}^{\prime}\right),
$$

$\lambda$ exprimant le rapport entre le couple atmosphérique et les deux couples de marées. En gardant le rapport $\mathrm{N} / \mathrm{N}^{\prime}$ égal à 3,4 (frottement des marées de Jeffreys, proportionnel au carré de la vitesse) et utilisant les valeurs précédentes pour $k$ et $n / n^{\prime}(4,82$ et 13,4$)$, il vient pour le rapport $\nu / \nu^{\prime}$ :

$$
\frac{\nu}{\nu^{\prime}}=\frac{30,69-59,0 . \lambda}{4,40-4,4 . \lambda}
$$

Spencer Jones remarque alors que pour $\lambda=0,4$, $y / \nu^{\prime}$ prend la valeur 2,7 en accord avec les résultats de Sitter. Pour $\lambda=0,52$, les résultats sont alors en accord avec ceux de Brouwer et Woerkom, où $v$ serait nul et $v^{\prime}$ positif.

d) Devant tout cela, il nous a paru possible d'approfondir les discussions en essayant de généraliser les raisonnements de Sir Spencer Jones et en mettant en évidence certaines combinaisons des équations précédentes, plus faciles à discuter que celles utilisées par les astronomes cités dont nous avons lu certains travaux. On a vu plus haut les difficultés introduites dans le rapport $v / v^{\prime}$, les essais d'interprétation par le couple atmosphérique, etc. Essayons de préciser tout cela et nous verrons mieux la sensibilité des hypothèses faites par les astronomes. Nous avons en effet trouvé aisément, d'abord :

$$
\begin{gathered}
3 \mathrm{~N}=\left(\frac{n}{n^{\prime}} \nu^{\prime}-\nu\right) \frac{2 \mathrm{Mm} c^{2}}{\mathrm{M}+m} \\
\mathrm{~N}+\mathrm{N}^{\prime}-\mathrm{N}^{\prime \prime}-\frac{n}{n^{\prime}} \frac{\nu^{\prime}}{k} \cdot \frac{2 \mathrm{Mm} c^{2}}{\mathrm{M}+m}
\end{gathered}
$$

Nous en avons tiré trois équations essentielles :

Première équation :

$$
\frac{\mathrm{C}}{d w} d t=-\frac{n}{n^{\prime}} \cdot \frac{2 \mathrm{Mm} c^{2}}{\mathrm{M}+m} \cdot \frac{v^{\prime}}{k}
$$

D'où le résultat remarquablement simple déjà trouvé en l'absence du couple de Holmberg et encore valable en présence de celui-ci : le ralentissement de la rotation de la Terre ne dépend que de la valeur d'accélération séculaire du Soleil, l'ensemble des influences des marées lunaires et solaires et de l'éventuel couple thermique se résumant dans le seul mouvement apparent du Soleil.

\section{Deuxième équation :}

Introduisons un nouveau paramètre $\mu$, défini par le rapport des couples de marées lunaires et solaires.

$\mathrm{N} / \mathrm{N}^{\prime}=\mu$; nous aurons entre $\lambda, \mu$ et le rapport $\nu / \nu^{\prime}$ la relation simple:

$$
\frac{n^{\prime}}{n} \cdot \frac{\nu}{\nu^{\prime}}=1-\frac{3 \mu}{k(1+\mu)(1-\lambda)}
$$

En fonction de $\lambda$, le rapport $\nu / v^{\prime}$ varie hyperboliquement, passant par la valeur zéro pour :

$$
\lambda_{0}=1-\frac{3 \mu}{k(1+\mu)} ;
$$

ce qui permet, en introduisant $\lambda_{0}$, d'écrire (II) sous la forme particulièrement simple :

$$
\frac{n^{\prime}}{n} \cdot \frac{\nu}{v^{\prime}}=\frac{\lambda_{0}-\lambda}{1-\lambda}
$$

$v / v^{\prime}$ peut donc prendre toutes les valeurs entre le maximum pour $\lambda=0$ et l'infini négatif pour $\lambda=1$. Toutes les valeurs données par les divers astronomes pour ce rapport peuvent ainsi être expliquées par $\lambda$ convenable. Par contre, pour que la vitesse de rotation de la Terre soit constante, il faut que $v^{\prime}$ soit nul, donc que $\lambda$ soit égal à l'unité.

\section{Troisième équation :}

Elle sera donnée par le calcul de la déperdition d'énergie mécanique:

$$
\begin{gathered}
-\frac{d \mathrm{E}}{d t}=\frac{2 \mathrm{Mm} c^{2}}{\mathrm{M}+m} \frac{n^{\prime}-n}{3} \frac{n}{n^{\prime}} \times \\
{\left[1+\frac{3\left(w-n^{\prime}\right)}{k\left(n^{\prime}-n\right)(1-\lambda)}\left(\nu^{\prime}-\nu\right)\right]}
\end{gathered}
$$

Eliminons y à l'aide de (II); il vient :

$$
\begin{gathered}
-\frac{d \mathrm{E}}{d t}=\frac{2 \mathrm{M} m}{\mathrm{M}+\frac{c^{2}}{m}} \frac{n}{n^{\prime}} \frac{n^{\prime}-n}{k} \times \\
{\left[\frac{w-n^{\prime}}{n^{\prime}-n}+\frac{\mu}{1+\mu}\right] \frac{v^{\prime}}{1-\lambda}}
\end{gathered}
$$

La déperdition d'énergie mécanique de la terre peu avec $\mu=\mathrm{N} / \mathrm{N}^{\prime}$. le rapport $\left(w-n^{\prime}\right) /\left(n^{\prime}-n\right)$ vaut 28,5 (voisin de $w / n^{\prime}$ naturellement) et par suite $d \mathbf{E} / d t$ varie quand $\mu$ varie, proportionnellement à la parenthèse; donc, quand on utilise successivement les deux hypothèses de frottement de Jeffreys, la variation de $d \mathrm{E} / d t$ est seulement de $3 \%$, ce qui est négligeable. Ceci n'était pas évident a priori.

Prenons comme base, à l'inverse des astronomes, ce qu'il paraît raisonnable de tirer des calculs de frottement des marées : cela nous 
conduira à partir de $d \mathrm{E} / d t$ et de $\mathrm{N} / \mathrm{N}^{\prime}=\bar{\mu}$ comme données, grâce à (III bis); nous en déduisons donc une valeur de $v_{0}^{\prime}$, donc de $v^{\prime} /(1-\lambda)$.

On résume alors nos résultats par les deux équations simples :

$$
\begin{gathered}
v^{\prime}=v_{0}^{\prime}(1-\lambda) \\
\nu=\left(n / n^{\prime}\right) v_{0}^{\prime}\left(\lambda_{0}-\lambda\right)
\end{gathered}
$$

Les deux accélérations séculaires de la Lune et du Soleil déduites des observations de marées dépendent donc linéairement de $\lambda$, c'est-à-dire de l'importance du couple thermique, car $\lambda_{0}$ ne dépend que de $\mu$.

Voici une application numérique : nous prendrons les deux valeurs de $\mu$ correspondant aux deux hypothèses de frottement et un frottement total de marées de 1,4 milliard de $\mathrm{kW}$. Avec les données astronomiques bien connues $k=4,82$,

\begin{tabular}{|c|c|c|c|c|c|c|c|c|}
\hline & \multirow{2}{*}{$\lambda_{0}$} & \multirow{2}{*}{$y_{0}$} & \multirow{2}{*}{$y_{0}^{\prime}$} & \multicolumn{5}{|c|}{ VALEUR DE $y / y^{\prime}$} \\
\hline & & & & $\lambda=0$ & 0,2 & 0,4 & 0,6 & 0,8 \\
\hline $\begin{array}{l}\mu=3,4 \ldots \ldots \\
\mu=5,1 \ldots \ldots\end{array}$ & $\begin{array}{l}0,515 \\
0,48\end{array}$ & $\begin{array}{l}4,5 \\
4,6\end{array}$ & $\begin{array}{l}0,62 \\
0,73\end{array}$ & $\begin{array}{l}7,2 \\
6,3\end{array}$ & $\begin{array}{l}5,25 \\
4,7\end{array}$ & $\begin{array}{l}2,58 \\
1,8\end{array}$ & $\begin{array}{l}-2,85 \\
-4,0\end{array}$ & $\begin{array}{l}-19,2 \\
-21,5\end{array}$ \\
\hline
\end{tabular}
$\left(n / n^{\prime}\right)=13,4$, on aura le tableau :
La figure suivante donne les variations avec $\lambda$ de $v / v^{\prime}$, y et $v^{\prime}$ pour les deux valeurs de $\mu$ retenues.

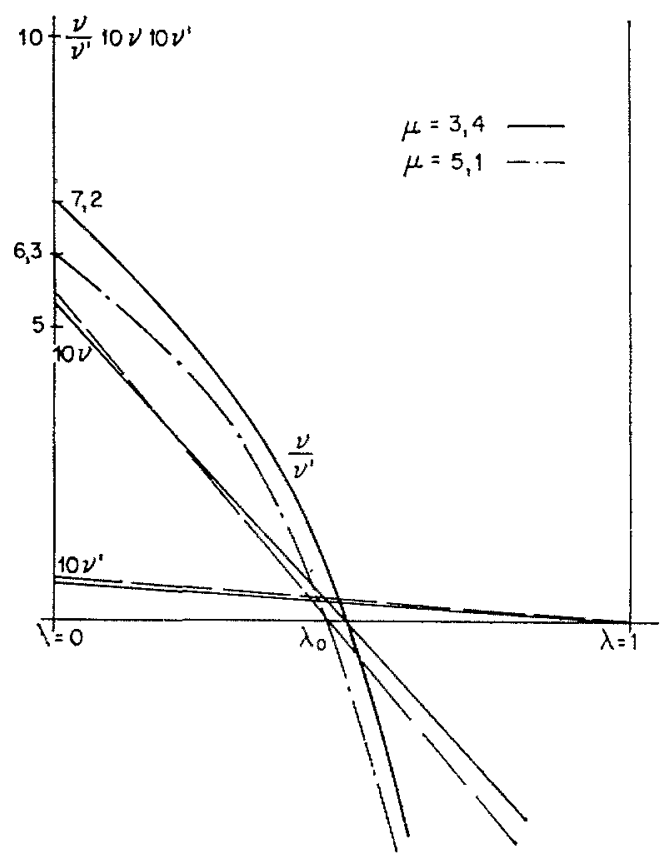

En conclusion, les accélérations v et v', que l'on peut chercher à estimer à partir des observations ou des calculs de marées et des couples correspondants, sont parfaitement compatibles avec le peu que nous pouvons tirer des observations astronomiques. Un bel édifice paraissait donc pouvoir être substitué à l'ancien trop désuet. L'énergie thermique du Soleil était substituée à l'énergie cinétique de la Terre par l'intermédiaire des marées atmosphériques, ceci par suite de l'identité entre la moitié de la période actuelle de rotation de la Terre et une des périodes propres d'oscillation de l'atmosphère. Il restait bien à ajuster les valeurs des deux couples pour que $\lambda$ égale l'unité, mais cela ne paraissait à personne un grand problème.

De mauvais esprits auraient pu, malgré ce succès du Soleil, faire remarquer qu'il n'est impressionnant que par sa masse, et non pas par son efficacité pour engendrer de l'énergie thermique, car une masse égale d'êtres humains respirant normalement produit cing mille fois plus de chaleur que le Soleil. Mais au total, la nouvelle théorie apparaissait belle.

Hélas! une petite note de l'article de Sir Spencer Jones renvoyait à un article de Kertz dont il nous faut maintenant parler...

\section{VI. - LES MAREES ATMOSPHERIQUES}

W. Kertz (10) est beaucoup moins affirmatif sur l'utilisation des marées atmosphériques et estime que les calculs de Holmberg ne sont pas convaincants du tout, car ils supposent une résonance atmosphérique très aiguë avec la période de douze heures, Résumons rapidement les informations rassemblées par Kertz; par leur sécheresse et leur précision elles feront un peu l'effet d'une douche froide : il existe indiscutablement des oscillations de la pression atmosphérique qui ne sont pas dues à l'attraction du Soleil et de la lune, mais au rayonnt- 
ment calorifique : la rotation de la Terre leur fournit a priori des périodes de un jour, $1 / 2$, $1 / 3,1 / 4$, etc.

La plus importante des oscillations observées a bien une période de 12 heures et est formẻe d'une onde se déplaçant avec le temps local (symbole $\mathrm{W}_{2}^{2}$ répétant les indices correspondants de développement en fonctions sphériques); son amplitude double à l'équateur atteint 3 millibar's, elle est symétrique par rapport à l'équateur, etc. On a observé aussi des ondes plus petites du même type (période 8 heures $\mathrm{W}_{4}{ }^{3}, \mathrm{~W}_{3^{3}}{ }^{3}, \mathrm{~W}_{5}{ }^{3}$ ) (périodes 6 heures $W_{4}^{4}, W_{5}^{4}$ ). Pratiquement, la période de 24 heures n'est pas observée.

Il faut ajouter à ces ondes qui suivent le mouvement du Soleil, une onde stationnaire $Z_{2}$ maximale au pôle et une onde lunaire $L_{2}$.

La phase $W_{2}^{2}$ démontre qu'elle ne peut être excitée seulement par des forces de gravitation. On comprendrait mal comment le terme $\mathrm{L}_{2}$ serait plus petit que le terme $W_{2}{ }^{2}$, car le terme de gravitation dù à la Lune est 2,2 fois plus grand que celui du Soleil. On doit donc supposer une excitation thermique due au rayonnement $\mathrm{du}$ Soleil, mais il faut alors expliquer pourquoi on ne peut pas observer une oscillation de période 24 heures. Lord Kelvin le premier, en 1882 comme nous l'avons vu, avança l'hypothèse d'une résonance et les calculs ont montré facilement qu'il ne peut en être question pour la période de 24 heures. Deux théories très différentes ont été présentées successivement. Une prenière théorie (1926) rend compte de $\mathrm{W}_{2}{ }^{2}$ par deux termes de grandeur voisine, l'un thermi- que, l'autre gravitationnel; mais l'amplification par résonance devrait alteindre un facteur de 100. De plus, une première difficulté réside dans le fait que pour expliquer la résonance la hauteur moyenne de l'atmosphère devrait ètre de $8 \mathrm{~km}$, alors que les observations de la propagation des ondes donnent pour cette valeur $10 \mathrm{~km}$ (explosion du Krakatau de 1883 par exemple). On peut s'en tirer en admettant que l'atmosphère possède une zone chaude entre 35 et $70 \mathrm{~km}$, ce qui introduit d'autres périodes de résonance, dont une correspondant à $8 \mathrm{~km}$ d'atmosphère normale. Malheureusement, si les mesures par fusées ont vérifié cette zone chaude (l'ozonosphère), l'élévation de température n'est pas suffisante pour retrouver le chiffre désiré de $8 \mathrm{~km}$. Enfin, difficulté supplémentaire, cette théorie n'expliquait pas les autres oscillations, en particulier $Z_{2}$.

Aussi, une deuxième théorie (1954) repose sur une excitation thermicque dépendant d'une variation de température indépendante de la hauteur: avec un modèle d'atmosphère très simple, il explique de façon suffisante des périodes de douze, huit et même six heures. L'effet de résonance joue aussi ici un certain rôle. Mais l'onde de 24 heures devrait être double de celle observée dans les meilleures conditions; Kertz estime donc que le problème n'est pas encore résolu de façon satisfaisante et que les conclusions d'Holmberg sur la vitesse de rotation de la Terre sont prématurées. Munk et MacDonald dans (11) parlent avec raison de la «jungle» des théories sur les marées atmosphériques.

\section{VII. - VARIATION DE LA CONSTANTE DE GRAVITATION}

Tous les problèmes précédents sont repris, au moins dans leur principe, dans le récent rapport (1960) du «Space Science Board of the National Academy of Sciences (U.S.A.) » consacré aux problèmes que les véhicules spatiaux pourraient aider à résoudre ou tout au moins à préciser. Ainsi, le deuxième chapitre est consacré entièrement à la gravitation et l'auteur R. H. Dicke s'y préoccupe des possibilités de constater une variation de la constante de gravitation G. D'après Newton, l'accélération due à une masse $m$ $\grave{a}$ distance $r$ est $\mathrm{Gm} / r^{2}$. Mach, dès 1872 , par des considérations dimensionnelles, avait montré que G est proportionnel à $\mathrm{R} / \mathrm{M}$, M étant la masse totale du monde visible supposée concentrée sur une coquille sphérique creuse de rayon $R$.

La cosmologie, admettant l'expansion générale du monde, conduit à $G$ variant en sens inverse de l'âge $T$ de l'univers, car $R$ croîtrait comme $\mathrm{T}$ et $\mathrm{M}$ comme $\mathrm{T}^{2}$. Il s'agit de grands problèmes, mais de petits effets, car la décrois- sance de $G$ à attendre est de 1 pour $10^{10}$ par an. Une variation séculaire de $G$ pourrait évidemment être mise en évidence par une observation précise de la vitesse de rotation de la Terre, comparant ainsi les mesures atomiques et gravitationnelles du temps. Car on peut espérer avoir une exactitude du temps atomique de 1 pour $10^{11}$. Si on vent útiliser des satellites, il faut obtenir le même ordre de grandeur de précision, soit 1 pour $10^{11}$, c'est-à-dire en gros la milliseconde de temps/an, ce qui est à porléc ou presque. Dans cette discussion, R. H. Dicke retrouve les problèmes précédents : détermination des accélérations sćculaires par des mesures modernes précises des mouvements de la Lune et du Soleil, utilisation des vieilles observations d'éclipses pour obtenir une relation linéaire entre les deux accélérations, etc.

Il cite en particulier une étude non publiée de W. H. Munk et C.F. E. MacDonald sur les actions et interactions, d'une part des marées 
Iunaires et solaires et d'autre part, de la marée solaire atmosphérique. Les auteurs croient pouvoir tenir correctement compte de l'effel des marées (ce qui suppose d'énormes progrès sur la situation telle que nous l'avons décrite) et ils trouvent une petite accélération résiduelle du mouvement de rotation de la Terre qui serait à peu près de l'ordre de grandeur et du bon signe pour représenter l'influence de la variation séculaire de la constante de gravitation. Mais Dicke fait remarquer qu'une baisse du niveau de la mer d'un mètre en mille ans accrô̂trait la vitesse de rotation de la Terre d'un montant égal en diminuant le moment d'inertie. De même, ajoute-t-il, l'interaction entre le champ magnétique de la Terre et le gaz ionise interplanétaire est mal connu. Il $\mathrm{y}$ a une foule de phénomènes mal connus qui peuvent donner des effets du même ordre de grandeur. Sa conclusion est donc qu'il y a peu de chance d'obtenir des informations sur la diminution de $\mathbf{G}$ par cette méthode. Nous nous associerons à cette conclusion.

Munk et MacDonald, dans leur préface (11), rappellent la phrase de Lord Kelvin en 1876 : «Troublé par les doutes de Newcomb sur les irrégularités de la Terre comme garde temps, je ne pouvais penser à rien d'autre que précision et nutation, marées et moussons, déplacement des régions équatoriales, fusion des glaces polaires». Il rentrait d'un voyage aux U.S.A. pour le discours traditionnel présidentiel devant une grande association britannique et devait parler des progrès scientifiques récents aux U.S.A., mais il ne parla que de la rotation de la Terre. Pour lui, pour Munk et MacDonald et pour nous, le sujet avait été irrésistible...

En résumé, la source de l'énergie des marées est-elle dans l'énergie cinétique de la Terre ou dans l'énergie thermique du Soleil? La belle image, par laquelle nous assimilions l'effet de l'œuvre de la Rance à un oiseau de paradis aux vives couleurs venant frotter de ses plumes une boule de diamant grande comme la Terre, estelle exacte? Avouons franchement qu'après tout ce tour d'horizon, nous n'en savons rien...

\section{DISCUSSION}

Sur la demande de M. le Président, M. Gougenherm fait connaître son point de vue sur l'exposé de M. GIBRAT. Il tient en premier lieu à féliciter M. GIBRát pour l'étude détaillée qu'il a faite d'une vaste documentation relative à un sujet très délicat, en apportant dans la discussion des résultats un esprit d'analyse pénétrant et un sens averti de la synthèse.

M. Gougenhem est d'accord avec M. Gibrat pour considèrer que, si les calculs de Jeffreys sur le frottement des marées sont très sujets à caution, ils doivent cependant conduire a un ordre de grandeur plausible. Il est regrettable que d'autres chercheurs n'aient pas repris ces calculs $a b$ ovo.

En ce qui concerne le mouvement de rotation de la Terre, M. Gougenheim pense que les travaux d'Holmberg et de Kertz constituent essentiellement le développernent d'hypothèses de travail et qu'ils garderont un caractère hypothétique tant qu'ils n'auront pas été confrontés avec succès à des faits d'observation. Dans l'état actuel de la question, les astronomes estiment que, jusqu'à notivel ordre, les travaux de Spencer Jones conservent toute leur valeur; l'autorité de Munk est loin d'être reconnue d'une manière aussi unanime.

L'accélération proportionnelle au moyen mouvement qui a été constatée dans les mouvements de révolution du Soleil et des planètes inférieures correspond bien à un ralentissement de la rotation terrestre. Pour les mouvements de la Lune, les phénomènes sont plus complexes : il faut considérer du point de vue mécanique le système Terre-Lune, et tenir compte de ce que les variations de la rotation de la Terre ont des répercussions sur le mouvement de la Lune.

La mise en service des horloges à quartz a permis aux astronomes de mettre en évidence des variations saisonnières de la vitesse de rotation de la Terre; celle-ci est tantôt en avance, tantôt en retard, de 2 ou 3 centièmes de seconde par rapport à une Terre moyenne. La cause en est d'ordre météorologique; les déplacements saisonniers des masses d'air (anticyclones d'hiver ou d'été) et des masses d'eaux (par exemple sous l'effet alternatif des moussons) font on effet varier le moment d'inertie de la Terre et influent par suite sur sa vitesse de rotation.

Les progrès réalisés récemment dans la mesure du temps, notamment par l'emploi des horloges atomiques qui sont d'ailleurs plus des étalons de fréquence que de véritables horloges, ont montré d'autre part que les manifestations importantes de l'activité solaire entrainent des changements brusques dans la vitesse de rotation de la Terre, qui est parfois accélérée, parfois ralentie. Le mécanisme par lequel un impetus est ainsi appliqué à la Terre reste encore très mal expliqué; on a songé $\grave{a}$ des couplages magnétiques, à des actions sur l'ionosphère, mais aucune théorie correcte n'a encore va le jour.

Enfin, il faut bien s'entendre sur ce qu'on appelle l'énergie des marées. Celle qui entretient le mouvement des eaux est d'origine gravitationnelle comme celle qui entretient les révolutions et les rotations des astres. Toutes les particules de l'univers ont été mises en mouvement à une certaine époque; elles se sont condensées en astres et en systèmes planètaires et les énergies en jeu dérivent de l'énergie initiale. Mais si l'on s'intéresse à l'énergie dissipée par les marées, c'est bien le frottement des eaux sur le fond et sur les parois des bassins océaniques qu'il faut prendre en considération; ce frottement entraine un ralentissement de la rotation terrestre et l'énergie dissipée est donc effectivement prélevée sur l'énergie cinétique de la rotation terrestre. Même si un autre phénomène venait compenser ce ralentissement, le frottement des marées n'en existerait pas moins avec une dissipation d'énergie cinétique qui serait aussitôt compensée par un rapport égal d'énergie d'une autre origine.

M. le Président conclut en soulignant l'intérêt général des questions relevées par la rotation de la Terre. Cette question fait l'objet d'un livre récent de 200 pages de Munk et MacDonald (Cambridge University Press, 1960), mais de nombreuses choses intéressantes et nouvelles peuvent encore être dites à ce sujet. 